\title{
ANHANG
}

\section{ITAS-Publikationen und Vorträge 2001}

\section{Monografien}

Coenen, R.; Fürniß, B.; Kupsch, Chr.: Technikfolgenabschätzung im Spiegel ihrer Institutionen - Eine Dokumentation über deutsche Forschungseinrichtungen auf dem Gebiet der Technikfolgenabschätzung. Karlsruhe: Forschungszentrum Karlsruhe, 2001

Grunwald, A.; Coenen, R.; Nitsch, J.; Sydow, A.; Wiedemann, P. (Hrsg.): Forschungswerkstatt Nachhaltigkeit - Wege zur Diagnose und Therapie von Nachhaltigkeitsdefiziten. Berlin: edition sigma Global zukunftsfähige Entwicklung-Perspektiven f. Deutschland, Bd. 2, 2001

Hennen, L.; Petermann, Th.; Sauter, A.: Das genetische Orakel. Prognosen und Diagnosen durch Gentests eine aktuelle Bilanz. Berlin: Edition Sigma. Studien des Büros für Technikfolgen-Abschätzung beim Deutschen Bundestag, Bd. 10, 2001

Kopfmüller, J.; Brandl, V.; Jörissen, J,; Paetau, M.; Banse, G.; Coenen, R.; Grunwald, A.: Nachhaltige Entwicklung integrativ betrachtet. Konstitutive Elemente, Regeln, Indikatoren. Berlin: edition sigma, Global zukunftsfähige Entwicklung-Perspektiven f. Deutschland, Bd. 1, 2001

Oertel, D.; Fleischer, T.: Brennstoffzellen-Technologie: Hoffnungsträger für den Klimaschutz. Technische, ökonomische und ökologische Aspekte ihres Einsatzes in Verkehr und Energiewirtschaft. Berlin: Erich Schmidt. Beiträge zur Umweltgestaltung, Bd. A 146, 2001

Revermann, Ch.; Hennen, L.: Das maßgeschneiderte Tier. Klonen in Biomedizin und Tierzucht. Berlin: Edition Sigma. Studien des Büros für Technikfolgen-Abschätzung beim Deutschen Bundestag, Bd. 9, 2001

Riehm, U.; Orwat, C.; Wingert, B.: Online-Buchhandel in Deutschland. Die Buchhandelsbranche vor der Herausforderung des Internet. Karlsruhe: Forschungszentrum Karlsruhe, 2001

\section{Sammelbände}

Coenen, R. (Hrsg.): Integrative Forschung zum globalen Wandel - Herausforderungen und Probleme. Frankfurt u. a.: Campus. Gesellschaft - Technik - Umwelt, 2001

Grunwald, A. (Hrsg.): ITAS 1999/2000. Jahrbuch des Instituts für Technikfolgenabschätzung und Systemanalyse (ITAS). Karlsruhe: Forschungszentrum Karlsruhe, 2001

\section{Aufsätze}

Arlt, A.; Leible, L.; Seifert, H.; Nieke, E.; Fürniß, B.: Processing of Sewage Sludge for Energetic Purposes A Challenge for Process Technology. In: Spanish Waste Club ORBIT Association (Hrsg.): Organic Recovery and Biological Treatment - Proceedings of the International Conference ORBIT 2001 on Biological Processing of Waste: product-oriented Perspective (Part II) Seville: ORBIT Association, 2001, p. 229-235

Banse, G.: Electronik Governance and Elektronik Government. Öffentliche Verwaltung und elektronische Medien. In: Fobel, P.; Banse, G.; Kiepas, A. (Hrsg.): Etika a informacna spolocnost. Banska Bystrica: 2001, S. 35-47 Banse, G.: Empfehlungen und Begründungen. In: Langenbach, C.J.; Ulrich, O. (Hrsg.): Elektronische Signaturen - Kulturelle Rahmenbedingungen einer technischen Entwicklung. Berlin u. a.: Springer, Wissenschaftsethik und Technikfolgenbeurteilung, Bd. 12, 2001, S. 15-29

Banse, G.: Erfinden im Spannungsfeld von Methodik, Heiristik und Kreativität. In: Banse, G.; Müller, H.-P. (Hrsg.): Johann Beckmann und die Folgen. Erfindungen - Versuch der historischen, theoretischen und empirischen Annäherung an einen vielschichtigen Begriff. Münster u. a.: Waxmann, Cottbuser Studien z. Geschichte v. Technik, Arbeit u. Umwelt, Bd. 17, 2001, S. 27-47

Banse, G.: Informationstechnische Sicherheit im Spiegel der aktuellen Risikodiskussion. VEDA, TECHNIKA, SPOLECNOST. Science, Technology, Society. Prag: Teorie Vedy, Theory of Science, X(XXIII)2-3, 2001, S. 75-91 Banse, G.: Odyssee im Cyberspace - Einblicke und Ausblicke. In: Bundesamt für Sicherheit in der Informationstechnik (Hrsg.): 2001 - Odyssee im Cyberspace ? Sicherheit im Internet! Ingelheim: SecuMedia, Tagungsband, 7. Deutscher IT-Sicherheitskongreß des BSI 2001, 2001, S. 445-455 
Banse, G.: Papierwelt und digitale Welt - Kulturelle Reflexionen über einen Umbruch. In: Langenbach, C.J.; Ulrich, O. (Hrsg.): Elektronische Signaturen -Kulturelle Rahmenbedingungen einer technischen Entwicklung. Berlin u. a.: Springer, Wissenschaftsethik und Technikfolgenbeurteilung, Bd. 12, 2001, S. 35-47

Banse, G.: Problemaufriss: Elektronische und digitale Signaturen. In: Langenbach, C.J.; Ulrich, O. (Hrsg.): Elektronische Signaturen - Kulturelle Rahmenbedingungen einer technischen Entwicklung. Berlin u. a.: Springer, Wissenschaftsethik und Technikfolgenbeurteilung, Bd. 12, 2001, S. 1-10

Banse, G.: Risiko - Technikfolgenabschätzung - Entscheidung. In: Caysa, V.; Seidel, H.; Wittich, D. (Hrsg.): Naturwissenschaftliches Weltbild und Gesellschaftstheorie - Entscheidungen im Spannungsfeld von Naturprozessen und humaner Lebensgestaltung. Schkeuditz: GNN Verlag Sachsen/Berlin GmbH, Texte zur Philosophie, Heft 9, 2001, S. 53-74

Banse, G.; Bechmann, G.: Interdisziplinäre Risikoforschung - Von der Risikoanalyse zum Risikomanagement. In: Allenspach, M. (Hrsg.): Integriertes Risiko-Management - Perspektiven einer chancenorientierten Unternehmensführung. St. Gallen: Institut für Versicherungswirtschaft der Universität St. Gallen, 2001, S. 15-40

Banse, G.; Bechmann, G.: Risiko - Semantik und Topoi transdisziplinärer Risikoforschung. VEDA, TECHNIKA, SPOLECNOST. Science, Technology, Society. Prag: Teorie Vedy. Theory of Science, X(XXIII)2-3, 2001, S. 5-56

Bareth, G.; Heincke, M.; Glatzel, S.: Soil-land-use-system approach to estimate nitrous oxide emissions from agricultural soils. Nutrient Cycling in Agroecosystems 60(2001), S. 219-234

Bechmann, G.: Paradigmenwechsel in der Wissenschaft ? - Anmerkungen zur problemorientierten Forschung. In: Grunwald, A. (Hrsg.): ITAS 1999/2000. Jahrbuch des Instituts für Technikfolgenabschätzung und Systemanalyse (ITAS). Karlsruhe: Forschungszentrum Karlsruhe, 2001, S. 93-112

Bechmann, G.: Risk and the Post-Modern Society. VEDA, TECHNIKA, SPOLECNODT. Science, Technology, Society. Prag: Teorie Vedy. Theory of Science, X(XXIII)2-3, 2001, S. 107-129

Bechmann, G.: Risk and the Postmodern Society. In: Zucker, B. (Hrsg.): Risiko-Dialog - Von der Idee zur konkreten Umsetzung. St. Gallen: Institut für Versicherungswirtschaft der Universität St. Gallen, 2001, S. 139-154

Bechmann, G.; Gloede, F.; Leßmann, E.: International Power Supply Policy and Globalisation of Research: The Example of Fusion Research Fusion Engineering and Design. 58-59(2001), S. 1091-1095

Bechmann, G.; Stehr, N.: The Uncertainties of Risk Communication in Knowledge Societies. In: Matthies, M.; Malchow, H.; Kriz, J. (Hrsg.): Integrative Systems Approaches to Natural and Social Dynamics. Berlin u. a.: Springer, 2001, S. 289-303

Brandl, V.; Jörissen, J.; Kopfmüller, J.; Paetau, M.: Das integrative Konzept: Mindestbedingungen nachhaltiger Entwicklung. In: Grunwald, A.; Coenen, R.; Nitsch, J.; Sydow, A.; Wiedemann, P. (Hrsg.): Forschungswerkstatt Nachhaltigkeit - Wege zur Diagnose und Therapie von Nachhaltigkeitsdefiziten. Berlin: edition sigma, Global zukunftsfähige Entwicklung-Perspektiven f. Deutschland, Bd. 2 , 2001, S. 79-102

Brüggemann, A.; Coenen, R.; Fleischer, T.; Karger, C.: Gesellschaftliche Rahmenszenarien. In: Grunwald, A.; Coenen, R.; Nitsch, J.; Sydow, A.; Wiedemann, P. (Hrsg.): Forschungswerkstatt Nachhaltigkeit - Wege zur Diagnose und Therapie von Nachhaltigkeitsdefiziten. Berlin: edition sigma, Global zukunftsfähige EntwicklungPerspektiven f. Deutschland, Bd. 2, 2001, S. 127-140

Böhle, K.; Rader, M.; Riehm, U.; Weber, A.: Technology Assessment and electronic Money - Between Consultancy and Oversight. In: Institut für Technikfolgenabschätzung und Systemanalyse; VDI/VDE-Technologiezentrum Informationstechnik (Hrsg.): Innovations for an e-Society. Challanges for Technology Assessment. Teltow: VDI/VDE, 2001, S. 1-11

Coenen, R.: Die Umsetzung des Leitbilds in nationalen Nachhaltigkeitsstrategien. In: Grunwald, A.; Coenen, R.; Nitsch, J.; Sydow, A.; Wiedemann, P. (Hrsg.): Forschungswerkstatt Nachhaltigkeit - Wege zur Diagnose und Therapie von Nachhaltigkeitsdefiziten. Berlin: edition sigma, Global zukunftsfähige Entwicklung-Perspektiven f. Deutschland, Bd. 2, 2001, S. 59-78

Coenen, R.; Brandl, V.; Jörissen, J.; Kopfmüller, J.; Sardemann, G.: Das System der Nachhaltigkeitsindikatoren. In: Grunwald, A.; Coenen, R.; Nitsch, J.; Sydow, A.; Wiedemann, P. (Hrsg.): Forschungswerkstatt Nachhaltigkeit - Wege zur Diagnose und Therapie von Nachhaltigkeitsdefiziten. Berlin: edition sigma, Global zukunftsfähige Entwicklung-Perspektiven f. Deutschland, Bd. 2, 2001, S. 103-126

Fleischer, T.; Fugger, W.-D.; Grunwald, A.; Karger, C.; Poppenborg, A.: Nachhaltigkeitspotenziale von Schlüsseltechnologien. In: Grunwald, A.; Coenen, R.; Nitsch, J.; Sydow, A.; Wiedemann, P. (Hrsg.): Forschungswerkstatt Nachhaltigkeit - Wege zur Diagnose und Therapie von Nachhaltigkeitsdefiziten. Berlin: edition sigma, Global zukunftsfähige Entwicklung-Perspektiven f. Deutschland, Bd. 2, 2001, S. 267-289

Gloede, F.: Partizipative Technikfolgenabschätzung in Europa. In: Grunwald, A. (Hrsg.): ITAS 1999/2000. Jahrbuch des Instituts für Technikfolgenabschätzung und Systemanalyse (ITAS). Karlsruhe: Forschungszentrum Karlsruhe, 2001, S. 186-192 
Grunwald, A.: Arbeitsteilige Technikgestaltung und verteilte Beratung: TA zwischen Politikberatung und Technikbewertung in Unternehmen. TA-Datenbank-Nachrichten 10(2001)2, S. 61-71

Grunwald, A.: Global zukunftsfähige Entwicklung - Perspektiven für Deutschland. In: Grunwald, A.; Coenen, R.; Nitsch, J.; Sydow, A.; Wiedemann, P. (Hrsg.): Forschungswerkstatt Nachhaltigkeit - Wege zur Diagnose und Therapie von Nachhaltigkeitsdefiziten. Berlin: edition sigma, Global zukunftsfähige Entwicklung-Perspektiven f. Deutschland, Bd. 2, 2001, S. 15-32

Grunwald, A.: HGF-Verbundprojekt „Global zukunftsfähige Entwicklung - Perspektiven für Deutschland“ eine Zwischenbilanz. TA-Datenbank-Nachrichten 10(2001)2, S. 82-90

Grunwald, A.: Integrative Forschung zum Globalen Wandel - Herausforderungen und Probleme. In: Coenen, R. (Hrsg.): Integrative Forschung zum Globalen Wandel - Herausforderungen und Probleme. Frankfurt u. a.: Campus, 2001, S.23-48

Grunwald, A.: The Application of Ethics to Engineering and the Engineer's Moral Responsibility: Perspectives for a Research Agenda. Science and Engineering Ethics 7(2001)3, p. 415-428

Grunwald, A.: The Relevance of Ethical Reflection for Technology Assessment: The Case of the Internet. In: Institut für Technikfolgenabschätzung und Systemanalyse; VDI/VDE-Technologiezentrum Informationstechnik (Hrsg.): Innovations for an e-Society. Challanges for Technology Assessment. Teltow: VDI/VDE, 2001, S. 1-8

Grunwald, A.: Vom Alten zum Neuen. Zur Planbarkeit technischer Erfindungen. In: Banse, G.; Müller, H.-P. (Hrsg.): Johann Beckmann und die Folgen. Erfindungen - Versuch der historischen, theoretischen und empirischen Annäherung an einen vielschichtigen Begriff. Münster u. a.: Waxmann, Cottbuser Studien z. Geschichte v. Technik, Arbeit u. Umwelt, Bd. 17, 2001, S. 277-291

Grunwald, A.: Warum das Thema „TA und Industrie“ umfunktionieren in eine Generaldiskussion über TA? - Eine Erwiderung auf Hauke Fürstenwerth. TA-Datenbank-Nachrichten, 10(2001)1, S. 153-155

Grunwald, A.: Warum integrative Forschung zum Globalen Wandel? In: Coenen, R. (Hrsg.): Integrative Forschung zum Globalen Wandel - Herausforderungen und Probleme, Frankfurt u. a.: Campus, Gesellschaft - Technik - Umwelt, 2001, S. 23-48

Grunwald, A.: Was macht den Abfall zum Abfall? Die Dauer der Abfalleigenschaft und Abschätzung ihrer ökologischen, ökonomischen und sozialen Folgen. In: Klett, W. (Hrsg.): Abfall ohne Ende? Oder: Ende der Abfalleigenschaft durch Umwandlung in Rohstoff. Köln: Gutke, 2001, S. 1-25

Grunwald, A.; Karger, C.: Nachhaltigkeit, Dialog und Stakeholder-Beteiligung. In: Grunwald, A.; Coenen, R.; Nitsch, J.; Sydow, A.; Wiedemann, P. (Hrsg.): Forschungswerkstatt Nachhaltigkeit - Wege zur Diagnose und Therapie von Nachhaltigkeitsdefiziten. Berlin: edition sigma, Global zukunftsfähige Entwicklung-Perspektiven f. Deutschland, Bd. 2, 2001, S. 171-186

Hennen, L.: TA in Biomedicine and Healthcare - From clinical Evaluation to policy Consulting. TA-DatenbankNachrichten 10(2001)1, S. 13-22

Jörissen, J.; Kneer, G.; Rink, D.: Wissenschaftliche Konzeptionen zur Nachhaltigkeit. In: Grunwald, A.; Coenen, R.; Nitsch, J.; Sydow, A.; Wiedemann, P. (Hrsg.): Forschungswerkstatt Nachhaltigkeit - Wege zur Diagnose und Therapie von Nachhaltigkeitsdefiziten. Berlin: edition sigma, Global zukunftsfähige EntwicklungPerspektiven f. Deutschland, Bd. 2, 2001, S. 33-58

Klann, U.; Schulz, V.: Die Aktivitätsfeldanalyse auf Basis von Input-Output-Tabellen. In: Grunwald, A.; Coenen, R.; Nitsch, J.; Sydow, A.; Wiedemann, P. (Hrsg.): Forschungswerkstatt Nachhaltigkeit - Wege zur Diagnose und Therapie von Nachhaltigkeitsdefiziten. Berlin: edition sigma, Global zukunftsfähige EntwicklungPerspektiven f. Deutschland, Bd. 2, 2001, S. 141-170

Kopfmüller, J.: Umsetzung des Nachhaltigkeitskonzepts im Energiebereich. In: Grunwald, A. (Hrsg.): ITAS 1999/2000. Jahrbuch des Instituts für Technikfolgenabschätzung und Systemanalyse (ITAS). Karlsruhe: Forschungszentrum Karlsruhe, 2001, S. 9-24

Krings, B.-J.: „,Co-Operation or Competition?“ New Models of Work Organisation in the Information Society. In: Institut für Technikfolgenabschätzung und Systemanalyse; VDI/VDE-Technologiezentrum Informationstechnik (Hrsg.): Innovations for an e- Society. Challanges for Technology Assessment. Teltow: VDI/VDE, 2001, S. 1-11

Krings, B.-J.: A Flexibilizacao do Trabalho Melhorara Realmente as Perspectivas de Carreira das Mulheres? ORGANIZACOES E TRABALHO (2001)25, S. 9-19

Krings, B.-J.: Ein Brot, das nur den halben Hunger stillt. Wechselwirkung 23(2001)110, S. 44-53

Leible, L.; Arlt, A.; Nieke, E.; Fürniß, B.: Energetic Use of organic Waste - Status and Potential for German Energy Supply. In: Spanish Waste Club ORBIT Association (Hrsg.): Organic Recovery and Biological Treatment - Proceedings of the International Conference ORBIT 2001 on Biological Processing of Waste: a productoriented Perspective (Part I). Seville: ORBIT Association, 2001, p. 129-136 
Leible, L.; Kälber, S.; Nieke, E.: Nachwachsende Rohstoffe - eine Zwischenbilanz. In: Grunwald, A. (Hrsg.): ITAS 1999/2000. Jahrbuch des Instituts für Technikfolgenabschätzung und Systemanalyse (ITAS). Karlsruhe: Forschungszentrum Karlsruhe, 2001, S. 25-41

Leible, L.; Nieke, E.; Arlt, A.; Fürniß, B.: Organic Wastes - The Biomass Resource with the greatest Importance for the Future. In: Kyritsis, S.; Beenackers, A.A.C.M.; Helm, P.; Grassi, A.; Ciaramonti, D. (Hrsg.): 1st World Conference on Biomass for Energy and Industry Proceedings (Volume 1). London: James \& James (Science Publishers) Ltd, 2001, p. 349-352

Meyer, R.; Petermann, Th.: Umwelt und Gesundheit - Bewertungskontroversen und Kommunikationsstrategien. TAB-Brief Nr. 20 / Juni 2001. (2001)20, S. 8-11

Meyer, R.; Petermann, Th.: Umwelt und Gesundheit - Bewertungskontroversen und Kommunikationsstrategien. In: Grunwald, A. (Hrsg.): ITAS 1999/2000. Jahrbuch des Instituts für Technikfolgenabschätzung und Systemanalyse (ITAS). Karlsruhe: Forschungszentrum Karlsruhe, 2001, S. 81-92

Nitsch, J.; Rösch, Ch.: Perspektiven für die Nutzung regenerativer Energien. In: Grunwald, A.; Coenen, R.; Nitsch, J.; Sydow, A.; Wiedemann, P. (Hrsg.): Forschungswerkstatt Nachhaltigkeit - Wege zur Diagnose und Therapie von Nachhaltigkeitsdefiziten. Berlin: edition sigma, Global zukunftsfähige Entwicklung-Perspektiven f. Deutschland, Bd. 2, 2001, S. 290-323

Oertel, D.; Fleischer, T.: Vergleichende Analyse zum Einsatz von Brennstoffzellen. GWF 142(2001)7, S. 486-492

Orwat, C.: Buchhandel und Internet - Zur These der Disintermediation durch den elektronischen Handel. In: Grunwald, A. (Hrsg.): ITAS 1999/2000. Jahrbuch des Instituts für Technikfolgenabschätzung und Systemanalyse (ITAS). Karlsruhe: Forschungszentrum Karlsruhe, 2001, S. 42-62

Orwat, C.; Riehm, U.; Wingert, B.: The Power of the Middleman in Electronic Markets - The Case of the German Bookselling Industry. In: Institut für Technikfolgenabschätzung und Systemanalyse; VDI/VDETechnologiezentrum Informationstechnik (Hrsg.): Innovations for an e- Society. Challanges for Technology Assessment. Teltow: VDI/VDE, 2001, S. 1-9

Paschen, H.; Coenen, Chr.; Wingert, B.: Neue Medien und Kultur - Medienentwicklung und kulturelle Transformation. TAB-Brief Nr. 21 / Dezember 2001. (2001)21, S. 5-9

Petermann, Th.: Technikkontroversen und Risikokommunikation. TAB-Brief Nr. 20 / Juni 2001. (2001)20, S. 5-7

Petermann, Th.; Hennen, L.; Sauter, A.: Risikodiskurse - ein Thema in neuen TAB-Projekten. TAB-Brief Nr. 20 / Juni 2001. (2001)20, S. 16-20

Pfitzmann, B.; Riordan, J.; Stüble, Ch.; Waidner, M.; Weber, A.: Die PERSEUS Systemarchitektur. In: Fox, D.; Köhntopp, M.; Pfitzmann, A. (Hrsg.): Verlässliche IT-Systeme - Sicherheit in komplexen IT-Infrastrukturen. Braunschweig / Wiesbaden: Vieweg \& Sohn, DuD-Fachbeiträge, 2001, S. 1-17

Riehm, U.: Einsatz elektronischer Diskussionsforen in Projekten zur Technikfolgenabschätzung. In: MaierRabler, U.; Latzer, M. (Hrsg.): Kommunikationskulturen zwischen Kontinuität und Wandel. Universelle Netzwerke für die Zivilgesellschaft. Konstanz: UVK, Schriftenreihe der Deutschen Gesellschaft für Publizistik- und Kommunikationswissenschaft, Bd. 28, 2001, S. 77-93

Rösch, Ch.; Heincke, M.: Ernährung und Landwirtschaft. In: Grunwald, A.; Coenen, R.; Nitsch, J.; Sydow, A.; Wiedemann, P. (Hrsg.): Forschungswerkstatt Nachhaltigkeit - Wege zur Diagnose und Therapie von Nachhaltigkeitsdefiziten. Berlin: edition sigma, Global zukunftsfähige Entwicklung-Perspektiven f. Deutschland, Bd. 2, 2001, S. 240-265

Sauter, A.: Risikomanagement transgener Pflanzen: Nachzulassungs-Monitoring als Lösung? TAB-Brief Nr. 20 / Juni 2001. (2001)20, S. 12-15

Sauter, A.; Petermann, Th.; Revermann, Chr.: Fortschritt und vermeintliche Grenzen - Moderne Biomedizin als Gegenstand von TA. In: Grunwald, A. (Hrsg.): ITAS 1999/2000. Jahrbuch des Instituts für Technikfolgenabschätzung und Systemanalyse (ITAS). Karlsruhe: Forschungszentrum Karlsruhe, 2001, S. 63-80

Seht, H. von: Global denken, lokal handeln und profitieren? Auswirkungen kommunaler Klimaschutzmaßnahmen. Raumforschung und Raumordnung 59(2001)2-3, S. 205-215

Seht, H. von: Lokaler Klimaschutz - was haben Kommunen davon? EILDIENST (2001)3, S. 31-33

Stelzer, V.: Stadt und Umwelt - Visionen 2030. Berichte zur deutschen Landeskunde, 75. Band, (2001)2/3, S. 315-319

Stelzer, V.; Jörissen, J.: Wohnen und Bauen. In: Grunwald, A.; Coenen, R.; Nitsch, J.; Sydow, A.; Wiedemann, P. (Hrsg.): Forschungswerkstatt Nachhaltigkeit - Wege zur Diagnose und Therapie von Nachhaltigkeitsdefiziten. Berlin: edition sigma, Global zukunftsfähige Entwicklung-Perspektiven f. Deutschland, Bd. 2, 2001, S. 218-239

Weber, A.: Sicherheit von elektronischen Zahlungssystemen. In: Adrian, L.; Siegfried, Chr. (Hrsg.): Einkaufen mit oder ohne Netz? 1. Werkstattbericht „E-Shopping und Kommunen“. Berlin: Deutsches Institut für Urbanistik, Materialien, Bd. 5, 2001, S. 66-77 
Weber, A.: Will Multi-Application Mobile Phones be Secure? L'AGEFI - FINANCE \& TECHNOLOGIE (2001)213, p. 54-56

Wingert, B.: Neue Medien und Kultur - Das Internet als kulturelles Gedächtnis? TAB-Brief Nr. 21 / Dezember 2001. (2001)21, S. 15-19

\section{Nicht publizierte Vorträge}

Achternbosch, M.; Bräutigam, K.-R.: Co-Incineration of Secondary Fuels in Cement Kilns. Lecture: Incineration 2001: 3rd international symposium on incineration. Brüssel, Belgien, July 2-4, 2001

Achternbosch, M.; Richers, U.: Comparison and Assessment of liquid Discharge and dry Discharge Flue Gas cleaning Systems of Municipal solid Waste Incinerators (MSWI). Lecture: IT3-Conference, Philadelphia, Pa., May 14-18, 2001

Achternbosch, M.; Richers, U.: Comparison of the Impacts of Sewage of Municipal Solid Waste Incinerators and Households by Model Calculations. Lecture: Incineration 2001: 3rd international symposium on incineration, Brüssel, Belgien, July 2-4, 2001

Banse, G.: Chancen und Gefahren der Informationstechnik. Vortrag am Istitut für Philosophie der Russischen Akademie der Wissenschaften, Moskau, Russland, 17. März 2001

Banse, G.: Der Informationsgesellschaft entgegen: ITA in Ländern Mittel- und Osteuropas. Vortrag im Rahmen des ITA-Gesprächskreises des BMBF, Karlsruhe, 7. Juni 2001

Banse, G.: Ethische Implikationen digitaler Signaturen. Vortrag beim Workshop „Technikethik und Informationsgesellschaft“" an der FernUniversität Hagen, 16. Februar 2001

Banse, G.: Informationsgesellschaft - Electronic Government - Ethik. Aktuelle Probleme. Vortrag auf dem Internationalen Workshop „Ethik und Informationsgesellschaft“, Humanwissenschaftliche Fakultät der Matej-BelUniversität Banská Bystrica Slovakia, 19. April 2001

Banse, G.: Johann Beckmann und die Folgen. Allgemeine Technologie in Vergangenheit und Gegenwart. Vortrag zum gemeinsam von ITAS und der Leibniz-Sozietät veranstalteten Symposium „Allgemeine Technologie Vergangenheit und Gegenwart“", Berlin, 12. Oktober 2001

Banse, G.: Neue Medien und Kultur. Vortrag im Rahmen des Internationalen Workshop „Internet Teaching and Online Learning - Experiences and Problems“, Universität Potsdam, 26. März 2001

Banse, G.: Neue Medien und Kultur. Aus der Arbeit an einem Projekt. Vortrag an der BTU Cottbus im Rahmen des Philosophischen Kolloquiums, Cottbus, 6. Juni 2001

Banse, G.: Technikfolgenabschätzung - Risiko - Entscheidung. Vortrag auf dem Kolloquium „Entscheidungen im Spannungsfeld von Naturprozessen und humaner Lebensgestaltung“, Rosa-Luxemburg-Stiftung Sachsen e.V., Leibnitz-Sozietät, 21. April 2001

Banse, G.: Über den Umgang mit Ungewissheit. Vortrag zur Konferenz „Rationalität heute - Vorstellungen, Wandlungen, Herausforderungen“", Ustron, Polen, 23.-25. September 2001

Banse, G.: Über den Umgang mit Ungewissheit. Vortrag im Institut für Philosophie der Akademie der Wissenschaften der Tschechischen Republik, Prag, Tschechische Republik, 13. Dezember 2001

Bechmann, G.: Basisanalyse Wandel von Kulturverständnis und Kulturkonzepte. Präsentation des TAB-Projekts „Neue Medien und Kultur“ vor dem Ausschuss „Kultur und Medien“ des Deutschen Bundestages, Berlin, 8. März 2001

Bechmann, G.: Globalisierung der Arbeit und der Einsatz von Informations- und Kommunikationstechnologien. Vortrag auf der Tagung „Evangelische Akademie Bad Herrenalb“, 26. Januar 2001

Bechmann, G.: Globalisierung, kulturelle Vielfalt und gesellschaftliche Rationalität. Vortrag auf der Konferenz „Rationalität heute - Vorstellungen, Wandlungen, Herausforderungen“ an der Universität Katowice, PL, 23.-25. Oktober 2001

Bechmann, G.: Mehr Risiko oder mehr Sicherheit durch Technik ? - Probleme der Technikfolgenabschätzung. Vortrag an der Medizinischen Universität Lübeck: Studium Generale, Lübeck, 17. Mai 2001

Bechmann, G.: Paradigmenwechsel in der Systemtheorie: von Bertalanffy zu Luhmann. Vortrag auf der internationalen Konferenz: „Bertalanffy heute“, Russische Akademie der Wissenschaften, Moskau, Russland, 29.-31. Oktober 2001

Bechmann, G.: Risk and the Development of Society. Lecture: Conferencia „El riesgo en las sociedades contemporaneas“, Universidad Valencia, E, December 6-8, 2001

Bechmann, G.: The Develpoment of the Information Society and Globalisation of Work - Information Technologies, new Forms of Work and Change in firm Culture. Lecture: Congreso international de tecnologics de la information y su repesubsion en la sociedad actual, Universidad Autoroma de Pueblo, Mexico, August 22-24, 2001 
Bechmann, G.: The cultural Function of the Internet - Pluralism and Rationality of Culture in a globalized World. Lecture: Congress „Innovations for an e-Society. Challanges for Technology Assessment“, Berlin, Germany, October 18, 2001

Bechmann, G.: Vom Subjekt zum autopoietischen System - Konstruktionsprobleme einer modernen Gesellschaftstheorie. Vortrag beim Soziologischen Seminar an der TU-Dresden, 11. Januar 2001

Bechmann, G.: Zukunft als Risiko oder Gefahr - Zur Bedeutung des Nichtwissens und der Sicherheit in der modernen Gesellschaft. Vortrag bei der Ringvorlesung an der Universität Bochum, 21. Januar 2001

Bechmann, G.; Beck, S.: E-Governance: Verwaltungsmodernisierung zwischen Demokratisierung und Rationalisierung - zur Verwendungsweise des Internets. Vortrag beim Forschungskolloquium: Staatsmodernisierung und E-Governance, Verwaltungshochschule Spayer, 5. Juli 2001

Beck, S.: Assessments in a corporate Culture: The German Experience. Lecture on the workshop „Localizing and Globalizing: Knowledge Cultures of Environment and Development“", Kennedy School of Government, Harvard University, Harvard, USA, April 6-7, 2001

Beck, S.: E-Democracy in e-Government: Citizen Participation in the Modernisation of public Administrations. Lecture: Congress „Innovations for an e-Society. Challanges for Technology Assessment“, Berlin, Germany, October 18, 2001

Beck, S.: The cultural Climate of Climate: The Role of political Culture in the German Response to Climate Change. Lecture on the 42nd annual Conference of the International Studies Association (ISA), Chicago, IL, USA, February 20-24, 2001

Brune, D.: Management of Biodegradable Waste between EU-Landfill, Energy Recovery and Soil Conservation. Lecture: Workshop on Pilot Projects in Environment Statistics Multi-Country PHARE Programme, Bled, Slovenia, June 18-22, 2001

Brune, D.: Organic Fertilisers from Sewage Sludge and Quality of Soil. Lecture: Contribution to the Conference „Sustainable Development - Forum for Partnership“, Malmö, S, June 27-29, 2001

Brune, D.: Waste from electric and electronic Equipment - Methods for Estimation of Amount, Content of various Materials Future reporting Obligations. Lecture: Workshop on Environment Statistics (Multi country PHARE programme), Budapest, Hungary, November 12-18, 2001

Bräutigam, K.-R.; Achternbosch, M.: Co-Incineration of Wastes in Cement Kilns-Mass Balances of selected Heavy Metals. Lecture: IT3-Conference, Philadelphia, Pa., May 14-18, 2001

Böhle, K.: Internet-Zahlungssysteme in Europa - Stand und Perspektive. Vortrag beim 5. IIR-Kongress, Zahlungssysteme im eBusiness, Frankfurt/Main, 7. Februar 2001

Coenen, R.: Fallstricke beim regionalen Vergleich von Nachhaltigkeit. Vortrag: Indikatoren-Workshop an der Universität Köln, „NZW im Dickicht der Nachhaltigkeitsindikatoren“, Köln, 7. September 2001

Coenen, R.; Krings, B.-J.: National $R \&$ D policies and Sustainable Development: Policy aims and strategic objectives. Vortrag beim Workshop: „Setting Concepts in Motion“(Veranstaltung des BMBF, IPTS, Europäische Kommission und ITAS), Bonn, 1. Februar 2001

Fleischer, T.: Brennstoffzellen-Technologie - Ausgewählte Ergebnisse einer TA-Studie für den Deutschen Bundestag. Vortrag auf der Frühjahrstagung der AK Energie der Deutschen Philosophischen Gesellschaft, Bad Honnef, 19.-20. April 2001

Fleischer, T.: Potentiale, Perspektiven und Probleme der Nanotechnologie. Vortrag: ZUFO/SOZF-Workshop „Sozial-ökologische Transformationsprozesse' und das Innovationsfeld der Bio- und Gentechnologie“, Rheine, 17.-18. Mai 2001

Fleischer, T.; Halbritter, G.: Innovation Strategies for Intelligent Transport Systems for a „Sustainable Mobility“. Lecture: 8th World Congress on Intelligent Transport Systems, Sydney, Australia, September 30 - October 4, 2001

Gorokhov, V. G.: Problems of Assessment of Scientific and Technological Development. Lecture: Conference: Critical Issues in Science and Technology Studies, University of Graz, A, June 2001

Grunwald, A.: Contributions of Technology Assessment for shaping e-Society. Lecture: Workshop on the Knowledge Society and European Foresight, Dublin, Ireland, December 12, 2001

Grunwald, A.: Die Adressatenfrage der Technikfolgenabschätzung. Vortrag beim ITA-Gesprächskreis, Forschungszentrum Karlsruhe, 7.-8. Juni 2001

Grunwald, A.: Die Ambivalenz von Innovationen und die Zukunft der Arbeit. Ethische Aspekte des elektronischen Handels. Vortrag: Tagung „Wirtschaftsethische Fragen der e-Economy“, Stuttgart, 15. November 2001

Grunwald, A.: Die Realisierung eines Nachhaltigen Konsums - Aufgabe der Konsumenten? Vortrag: Fachkonferenz „Nachhaltiger Konsum“, Stuttgart/Hohenheim, 30. November 2001

Grunwald, A.: Ethische Aspekte der Folgenforschung. Vortrag: Symposium „Folgen von Folgenforschung“, Speyer, 16. November 2001 
Grunwald, A.: Interdisziplinäre Forschung in der Praxis. Barrieren und Brücken. Vortrag: DFG-Rundgespräch „Interdisziplinarität und Umweltwissenschaften“, Universität Trier, 9. November 2001

Grunwald, A.: Modellieren als Praxis. Normative Implikationen von Modellierungen und Modelltransfers. Vortrag am Philosophischen Institut der Universität Marburg, 10. Januar 2001

Grunwald, A.: Nachhaltige Gestaltung von Technik - Herausforderungen und Probleme. Vortrag am Zentrum für Interdisziplinäre Technikforschung der TU Darmstadt, 5. Dezember 2001

Grunwald, A.: Normative Implikationen von Modellierungen und Modelltransfers. Vortrag am Institut für Philosophie, Universität Marburg, 30. Mai 2001

Grunwald, A.: Partizipative Technikfolgenabschätzung und das Problem der Legitimation. Vortrag beim UFZ Leipzig, 8. Februar 2001

Grunwald, A.: Rationalität in der gesellschaftlichen Gestaltung der Technik? Vortrag: Kongress „Rationalität in Wissenschaft und Technik“, Wisla, Polen, 24. September 2001

Grunwald, A.: Technik nachhaltig gestalten? Vortrag auf der Konferenz „Innovationen für eine Nachhaltige Entwicklung“, Berlin, 5. November 2001

Grunwald, A.: Verantwortung im Netz. Ethische Fragen zum Umgang mit dem Internet. Vortrag beim Workshop „Technikethik und Informationsgesellschaft“ an der FernUni Hagen, 16. Februar 2001

Grunwald, A.: Wenn Roboter planen - begriffliche und andere Probleme handelnder Artefakte. Vortrag: Tagung der Sektion „Wissenschafts- und Technikforschung“ der Deutschen Gesellschaft für Soziologie, Berlin, 4. Oktober 2001

Halbritter, G.: Verkehrspolitische Handlungsoptionen - Wirksamkeit und Folgen. Vortrag auf der Tagung „Raus aus der Sackgasse - Perspektiven zukünftiger Verkehrspolitik“ in der Evangelischen Akademie Bad Boll, 26.-28. Januar 2001

Halbritter, G.; Fleischer, T.; Kupsch, Chr.: European and US Experiences with intelligent Transport Systems in metropolitan Areas with Respect to a more efficient and environmentally Sounder Transport System. Lecture: 8th World Congress on intelligent Transport Systems, Sydney, October 1, 2001

Kopfmüller, J.: Die ökonomische Dimension nachhaltiger Entwicklung. Vortrag im Rahmen der Vorlesungsreihe „Nachhaltigkeit im Bauwesen“ des Instituts für Industrielle Bauproduktion an der Universität Karlsruhe, 6. November 2001

Krings, B.-J.: „, Vagabundierendes Denken“ - Frauenforschung im Spannungsfeld von Wissenschaft und Gesellschaft. Vortrag am Forschungszentrum Karlsruhe, 12. November 2001

Krings, B.-J.: Individualisierung der Arbeit - Neue Arbeitskonzepte in der Informationsgesellschaft. Vortrag auf der Tagung: Wirtschaftsethische Fragen der E-Economy, Ausschuss Wirtschaftsethik der allgemeinen Gesellschaft für Philosophie in Deutschland, Stuttgart, 15.-16. November 2001

Krings, B.-J.; Moniz, A.: Konkurrenz oder Kooperation? Die sozio-kulturelle Entwicklung der Informationsgesellschaft. Vortrag beim XIV International Sociological Seminar: „Democracy and Participation in Organizations in the New international Societies“, San Juan, Argentina, June 27-30, 2001

Moniz, A. B.; Van Hootegem, G.; Krings, B.-J.: Technological Practices in the European Auto Industry: Exploring Cases from Belgium, Germany and Portugal. Lecture: IX th GERPISA International Colloquium, Université d'Evry-Val-d'Essonne, Paris, France, June 2001

Paskaleva-Shapira, K.: Innovative Partnerships for Sustainable Urban Tourism - Framework Approach and the European Experience. Conference Presentation: TTRA European Conference „Creating and Managing Growth in Travel and Tourism“, Sweden, April 20-25, 2001

Paskaleva-Shapira, K.: Promoting Partnerships for effective Governance of Sustainable Urban Tourism. The Case of Germany. Lecture: INTA. International Seminar „Tourism in the City - Opportunity for Regeneration and Development, Turin, Italy, February 20-22, 2001

Riehm, U.: Alles anders? Zum Handel mit digitalen Gütern am Beispiel der Musik- und Buchbranche. Vortrag auf dem 2. Fachgespräch in der TAB-Veranstaltungsreihe „Innovationsbedingungen des E-Commerce“, Berlin, 4. April 2001

Riehm, U.: Der Buchhandel vor der Herausforderung des Internets - Ergebnisse aus dem Projekt OnlineBuchhandel (POB). Vortrag auf dem Workshop „Online-Buchhandel“ der IHK Region Stuttgart, 9. Juli 2001

Riehm, U.: Digitalisierung und Individualisierung von Produkten und Diensten - Veränderungen in der Hersteller-Konsumenten-Beziehung. Vortrag auf der vierten Tagung des Ausschusses Wirtschaftsethik der Allgemeinen Gesellschaft für Philosophie in Deutschland „Wirtschaftsethische Fragen der E-Economy“, Stuttgart, 15.-17. November 2001

Weber, A.: Sicherheit und Zahlungssysteme. Vortrag beim Workshop des Deutschen Instituts für Urbanistik, Leipzig, 2. April 2001 
Weber, A.: Typische Abläufe bei der Nutzung digitaler Signaturen. Vortrag: Bundesamt für Sicherheit in der Informationstechnik, Bonn, 19. September 2001

Weber, A.: e-Payment Systems in Europe. Lecture: Digital Economy Development in South East Europe, Bucharest, R, October 4-5, 2001

\section{Forschungsberichte}

Halbritter, G.; Bräutigam, K.-R.; Fleischer, T.; Fulda, E.; Georgiewa, D.; Klein-Vielhauer, S.; Kupsch, Chr.: Verkehr in Ballungsräumen - Optionen für eine effizientere und umweltverträglichere Gestaltung. Karlsruhe: Forschungszentrum Karlsruhe, Wissenschaftliche Berichte, FZKA 6678, 2001

Hennen, L.: „Folgen von Umwelt- und Ressourcenschonung für Ausbildung, Qualifikation und Beschäftigung “ Vorstudie. Karlsruhe: Forschungszentrum. TAB-Arbeitsbericht Nr. 71 (Mai 2001), 2001

Klein-Vielhauer, S.: Neue Konzepte für den Wirtschaftsverkehr in Ballungsräumen. Ein Werkstattbericht über Bemühungen in Praxis und Wissenschaft. Karlsruhe: Forschungszentrum Karlsruhe, Wissenschaftliche Berichte, FZKA 6599 (Mai 2001), 2001

Oertel, D.; Fleischer, T.: Brennstoffzellen-Technologie - Endbericht. Karlsruhe: Forschungszentrum. TABArbeitsbericht Nr. 67 (Januar 2001), 2001

Paschen, H.; Banse, G.; Coenen, Chr.; Wingert, B.: Neue Medien und Kultur - Bisherige und zukünftige Auswirkungen der Entwicklung Neuer Medien auf den Kulturbegriff, die Kulturpolitik, die Kulturwirtschaft und den Kulturbetrieb - Vorstudie. Karlsruhe: Forschungszentrum, TAB-Arbeitsbericht Nr. 74, 2001

Riehm, U.; Orwat, C.; Wingert, B.: Online-Buchhandel in Deutschland. Die Buchhandelsbranche vor der Herausforderung des Internet. Stuttgart: Akademie für Technikfolgenabschätzung in Baden-Württemberg, Arbeitsbericht Nr. 192, Juni 2001, 2001 